\title{
THE EXOTIC (HIGHER ORDER LÉVY) LAPLACIANS GENERATE THE MARKOV PROCESSES GIVEN BY DISTRIBUTION DERIVATIVES OF WHITE NOISE
}

\author{
LUIGI ACCARDI \\ Centro Vito Volterra, Università di Roma "Tor Vergata", \\ 00133 Via Columbia 2, Roma, Italy \\ accardi@volterra.uniroma2.it \\ UN CIG JI* \\ Department of Mathematics, \\ Research Institute of Mathematical Finance, \\ Chungbuk National University, \\ Cheongju 361-763, Korea \\ uncigji@chungbuk.ac.kr \\ KIMIAKI SAITÔ ${ }^{\dagger}$ \\ Department of Mathematics, \\ Meijo University, \\ Nagoya 468, Japan \\ ksaito@meijo-u.ac.jp \\ Received 27 May 2013 \\ Published 20 September 2013 \\ Communicated by O. Smolyanov
}

\begin{abstract}
We introduce, for each $a \in \mathbb{R}_{+}$, the Brownian motion associated to the distribution derivative of order $a$ of white noise. We prove that the generator of this Markov process is the exotic Laplacian of order $2 a$, given by the Cesàro mean of order $2 a$ of the second derivatives along the elements of an orthonormal basis of a suitable Hilbert space (the Cesàro space of order $2 a$ ). In particular, for $a=1 / 2$ one finds the usual Lévy Laplacian, but also in this case the connection with the $1 / 2$-derivative of white noise is new. The main technical tool, used to achieve these goals, is a generalization of a result due to Accardi and Smolyanov ${ }^{5}$ extending the well-known Cesàro theorem to higher order arithmetic means. These and other estimates allow to prove existence of the heat
\end{abstract}

\footnotetext{
*This work was partially supported by NRF-JSPS Joint Research Project 2010 "Non-commutative Harmonic Analysis with Applications to Real World Complex Phenomena".

${ }^{\dagger}$ This work was supported by JSPS Grant-in-Aid Scientific Research 24540149 and Japan-Korea Basic Scientific Cooperation Program (2013-2015) "Non-commutative Stochastic Systems: Analysis, Modeling and Applications".
} 
semi-group associated to any exotic Laplacian of order $\geq 1 / 2$ and to give its explicit expression in terms of infinite dimensional Fourier transform.

Keywords: Exotic Laplacians; derivatives of white noise; Cesàro mean of higher order.

AMS Subject Classification: $60 \mathrm{H} 40$

\section{Introduction}

Essentially infinite dimensional Laplacians are Laplacians which are identically zero on all functions depending only on a finite number of variables (cylindric functions). The first example of such an operator was the Lévy Laplacian, defined by Lévy, ${ }^{21}$ as the Cesàro mean of the second derivatives, along a sequence of orthogonal vectors in a Hilbert space, while the usual Volterra Laplacian is defined by the series of these derivatives. An infinite hierarchy of operators with this property (more precisely, with the property that elements of higher rank are identically zero on the domain of elements of lower rank) was introduced by Accardi and Smolyanov ${ }^{3}$ in terms of higher order Cesàro means and called exotic Laplacians. However, while in Ref. 3, an explicit construction of the heat semi-group associated to the usual Levy Laplacian was obtained using ergodic theory techniques, these techniques could not be applied to generic exotic Laplacians and for them, the problem remained open until recently (see discussion below). In Refs. 1 and 16, it was proved that the Lévy Laplacian can be considered as a particular Volterra-Gross Laplacian. In Ref. 6, it was proved that all the exotic Laplacians can also be considered as particular Volterra-Gross Laplacians and generate infinite dimensional Brownian motions. A similarity transform relating any two Laplacians of the exotic hierarchy was given in Ref. 7 and it allowed to identify the exotic Laplacians of order $2 p+1(p \in \mathbb{N})$ with the generators of the (infinite dimensional) Brownian motion corresponding to the $p$ th distribution derivative of the standard Brownian motion.

The main purpose of this paper is to remove this restriction to odd integers. This goal is achieved through the proof of an improvement of the Accardi-Smolyanov theorem generalizing Cesàro theorem on arithmetic means of converging sequences (see Sec. 5, Ref. 5). We prove that the white noise generated by the exotic Laplacian of order $2 a\left(a \in \mathbb{R}_{+}\right)$is precisely the ath distribution derivative of the standard white noise and that the Lévy Laplacian corresponds to the derivative of order $1 / 2$ (see Sec. 3 for definitions). The paper is organized as follows.

In Sec. 2 we recall some basic notions and results of white noise analysis in a language that prepares the ground for the applications in the following section.

In Sec. 3 we prove that the second quantization of any positive power of the adjoint of the derivative operator, defined through the spectral theorem, gives an isomorphism from the space of white noise distributions onto itself. These isomorphisms map white noise functionals into functionals of higher order derivatives of white noise.

In Sec. 4, we study the higher order Cesàro mean and then prove the above mentioned generalization of the Accardi-Smolyanov result. ${ }^{5}$ 
In Sec. 5 we introduce exotic Laplacians and study their mutual relationships.

In Sec. 6 we construct the Cesàro spaces and prove their connections with derivatives of white noise. These results are used to show how the isomorphisms, given by second quantization of powers of the derivative operator, intertwine the actions of exotic Laplacians on a suitable domain of white noise distributions.

In Secs. 7 and 8, we extend the construction of Gel'fand triples to higher derivatives of white noise and introduce an embedding of test functionals for these triples into the space of test functionals for standard white noise distributions, which induces an embedding of the corresponding Cesàro spaces into the space of standard white noise distributions.

This embedding plays a crucial role in the construction of the Brownian motions generated by exotic Laplacians and associated heat semi-groups accomplished in the last Sec. 9, where we also prove the identification between the ath derivative of the Brownian motion generated by the Lévy Laplacian obtained in Ref. 16 with the Brownian motion associated with the exotic Laplacian of order $2 a$.

\section{Preliminaries}

\subsection{Test function spaces}

Let $H$ be a separable complex Hilbert space with inner product $(\cdot, \cdot)_{H}$ and with a conjugation operator $J: H \rightarrow H$ (antilinear, $\left.J^{2}=\mathrm{id},(J \xi, J \eta)_{H}=(\eta, \xi)_{H}\right)$. Unless otherwise specified, "operator" means "linear operator". Thus

$$
(J \cdot, \cdot)_{H}=:\langle\cdot, \cdot\rangle
$$

is a complex bilinear form on $H \times H$ and the inner product $(\cdot, \cdot)_{H}$ on $H$ is given by $(\cdot, \cdot)_{H}=:\langle J \cdot, \cdot\rangle$. The fixed point space of $J$, which is a real vector sub-space of $H$, is denoted $H_{\mathbb{R}}$ and one can prove that any orthonormal basis of $H_{\mathbb{R}}$ as a $\mathbb{R}$-vector space is an orthonormal basis of $H$ as a $\mathbb{C}$-vector space.

Let $A$ be a densely defined self-adjoint operator on $H$ with pure point simple spectrum such that

$$
\begin{aligned}
|A| & >1, \\
A J & =J A, \\
\operatorname{Tr}\left(A^{-2 p}\right) & <+\infty ; \quad \forall p \geq 1,
\end{aligned}
$$

where, for any $p \in \mathbb{R}$, the powers $A^{p}$ are defined by the spectral theorem.

Lemma 2.1. If $A$ satisfies (2.3) and

$$
A:=\sum_{k=1}^{\infty} \ell_{k} e_{k} e_{k}^{*} ; \quad e_{k} e_{k}^{*}(\xi):=\left\langle e_{k}, \xi\right\rangle e_{k} ; \quad \xi \in H
$$

is its spectral decomposition, then up to multiplication of each $e_{k}$ by a complex number of modulus 1, one can suppose that

$$
J e_{k}=e_{k} ; \quad \forall k \in \mathbb{N}^{*} .
$$


Proof. Since $J$ commutes with $A$ and the eigenvalues of $A$ are real then, $\forall k \in$ $\mathbb{N}, J e_{k}$ is an eigenvector of $A$ belonging to the same eigenvalue of $e_{k}$. Since the spectrum of $A$ is simple, $J e_{k}$ must be a multiple of $e_{k}$, i.e.,

$$
J e_{k}=u_{k} e_{k} .
$$

Since $J$ is an isometry, one must have $\left|u_{k}\right|=1$. Moreover, $\forall k \in \mathbb{N}^{*}$

$$
J\left(1+u_{k}\right) e_{k}=J e_{k}+J u_{k} e_{k}=u_{k} e_{k}+\overline{u_{k}} J e_{k}=u_{k} e_{k}+\left|u_{k}\right|^{2} e_{k}=\left(1+u_{k}\right) e_{k} .
$$

Therefore the vectors

$$
f_{k}:=\frac{1+u_{k}}{\left|1+u_{k}\right|^{1 / 2}} e_{k}
$$

form an orthonormal basis and one has

$$
J f_{k}=f_{k} .
$$

Since replacing each $e_{k}$ by $f_{k}$ with $\left|u_{k}\right|=1$ does not change the spectral decomposition of $A$, the thesis follows.

Remark 2.1. The numeration of the basis $e \equiv\left(e_{k}\right)$ of eigenvectors of $A$ can always be chosen so that

$$
1<\left|\ell_{1}\right|<\left|\ell_{2}\right|<\left|\ell_{3}\right|<\cdots
$$

and condition (2.3) becomes

$$
\sum_{k=1}^{\infty} \ell_{k}^{-2 p}<\infty ; \quad \forall p \geq 1
$$

Given $p \in \mathbb{R}$, if $p>0$, then $A^{p}$ is densely defined because its domain contains the dense sub-space of $H$ :

$$
E^{(0)}:=\text { algebraic linear span of }\left\{e_{k}: k \in \mathbb{N}^{*}\right\} .
$$

If $p \leq 0$, then $A^{p}$ is a contraction on $H$. Moreover, for any $p \in \mathbb{R}, E^{(0)}$ is an invariant sub-space for $A$ hence for all its powers. In particular for any $p \in \mathbb{R}$, the restriction of $A^{p}$ on $E^{(0)}$ is an invertible operator with inverse given by the restriction of $A^{-p}$ on $E^{(0)}$. For $p \in \mathbb{R}$ the scalar product

$$
(\xi, \eta)_{p}:=\left(A^{p} \xi, A^{p} \eta\right)_{0}:=\left(A^{p} \xi, A^{p} \eta\right)_{H}
$$

is well defined for $\xi$ and $\eta$ in the domain of $A^{p}$, nondegenerate and the associated $p$-norm

$$
|\xi|_{p}^{2}:=\left|A^{p} \xi\right|_{0}^{2}=\sum_{k=1}^{\infty} \ell_{k}^{2 p}\left|\alpha_{k}\right|^{2} ; \quad \xi=\left\{\alpha_{k}\right\}_{k=1}^{\infty}=\sum_{k=1}^{\infty} \alpha_{k} e_{k} \in H
$$


Exotic Laplacians Generate Markov Processes by Distribution Derivatives of White Noise

where $|\cdot|_{0}$ is the norm of $H$, is increasing as a function of $p$ in the sense that

$$
p, q \in \mathbb{R}, \quad p \leq q \Rightarrow|\xi|_{p} \leq|\xi|_{q} ; \quad \forall \xi \in H .
$$

One can prove that, for $p \geq 0$ the subspace of $H$

$$
E_{p}:=\left\{\xi \in H:|\xi|_{p}<\infty\right\}
$$

is closed under the norm $|\cdot|_{p}$, i.e., it is a Hilbert space for the scalar product (2.9).

For $p \geq 0$ we denote $E_{-p}$ the completion of $H$ with respect to the norm $\left.|\cdot|\right|_{-p}$.

Lemma 2.2. (i) For all $p>0$ the map

$$
V_{p}:\left(E_{p},(\cdot, \cdot)_{p}\right) \ni \xi \mapsto A^{p} \xi \in H
$$

is a partial isometry whose range is considered as a sub-space of $H$.

(ii) For all $p>0$ the map

$$
V_{-p}: H \ni \xi \mapsto A^{p} \xi \in\left(E_{-p},(\cdot, \cdot)_{-p}\right)
$$

is a partial isometry with dense range.

(iii) Defining $V_{0}: H \rightarrow H$ to be the identity map, for any $p \in \mathbb{R}$ the restriction of $V_{p}^{*}$ on the range of $V_{p}$ coincides with $A^{-p}$.

Proof. If $p>0$, then the map (2.11) satisfies

$$
\left(A^{p} \xi, A^{p} \eta\right)_{H}=(\xi, \eta)_{p}
$$

hence it is a partial isometry. Let $V_{p}^{*}: H \rightarrow\left(E_{p} \cdot(\cdot, \cdot)_{p}\right)$ denote its adjoint. If $\xi_{H} \in$ $V_{p} E_{p}$ (range of $V_{p}$ ), then there exists $\xi_{p} \in E_{p}$ such that $\xi_{H}=A^{p} \xi_{p}$. For this $\xi_{p}$ and for any $\eta_{p} \in E_{p}$ one has

$$
\left(V_{p}^{*} \xi_{H}, \eta_{p}\right)_{p}=\left(\xi_{H}, V_{p} \eta_{p}\right)_{H}=\left(\xi_{H}, A^{p} \eta_{p}\right)_{H}=\left(A^{p} \xi_{p}, A^{p} \eta_{p}\right)_{H}=\left(\xi_{p}, \eta_{p}\right)_{p} .
$$

Thus

$$
V_{p}^{*} \xi_{H}=V_{p}^{*}\left(A^{p} \xi_{p}\right)=\xi_{p}=A^{-p} \xi_{H}
$$

or equivalently

$$
\left.V_{p}^{*}\right|_{\text {Range } V_{p}}=A^{-p} \text {. }
$$

If $\xi_{H}$ is orthogonal to the range of $V_{p}$, then $V_{p}^{*} \xi_{H}=0$. If $p>0$, then by construction

$$
V_{-p}: H \rightarrow E_{-p}
$$

is a partial isometry with dense range. To calculate its adjoint, let $\xi_{-p} \in \operatorname{Range} V_{-p}$. Then there exists $\xi_{H} \in H$ such that $V_{-p} \xi_{H}=\xi_{-p}$. Therefore, for any $\eta_{H} \in H$ one has

$$
\begin{aligned}
\left(V_{-p}^{*} \xi_{-p}, \eta_{H}\right)_{H} & =\left(\xi_{-p}, V_{-p} \eta_{H}\right)_{-p}=\left(V_{-p} \xi_{H}, V_{-p} \eta_{H}\right)_{-p} \\
& =\left(A^{p} \xi_{H}, A^{p} \eta_{H}\right)_{-p}=\left(\xi_{H}, \eta_{H}\right)_{H} .
\end{aligned}
$$

Therefore

$$
V_{-p}^{*} \xi_{-p}=\xi_{H}=A^{-p} A^{p} \xi_{H}=A^{-p} V_{-p} \xi_{H}=A^{-p} \xi_{-p}
$$


In this case the orthogonal of the range of $V_{-p}$ is zero because this range is dense.

The family of Hilbert spaces

$$
\left\{\left(E_{p},(\cdot, \cdot)_{p}\right): p \in \mathbb{R}\right\}
$$

depending on the triple $\left(H,\left\{e_{k}\right\},\left\{\ell_{k}\right\}\right.$ ), (or equivalently on the pair $(H, A)$ ) is decreasing by inclusion and defines a pair of topological vector spaces through the relations:

$$
E:=\underset{p \rightarrow+\infty}{\operatorname{proj} \lim } E_{p} \subset E_{0}=H \subset E^{*}:=\operatorname{ind}_{p \rightarrow+\infty} \lim _{-p}
$$

Notice that for the orthonormal basis $e=\left\{e_{k}\right\}$ of $H$, defining $A, A^{-p} e=\left\{A^{-p} e_{k}\right\}$ is also an orthogonal basis of $\left(E_{p},(\cdot, \cdot)_{p}\right)$ for any $p \in \mathbb{R}$. In particular $E^{(0)}$ is a dense sub-space of each $E_{p}(p \in \mathbb{R})$.

The elements of $E$ are those elements of $H$ that are in the domain of $A^{p}$ for each $p \in \mathbb{R}$. Continuity of a linear operator $B$ in the projective topology on $E$ means that for any $p \in \mathbb{R}$ there exists some $q \geq 0$ and a constant $C_{p, q} \geq 0$ such that for all $p \in \mathbb{R}$ there exists a constant $C_{B, p}$ such that

$$
|B \xi|_{p} \leq C_{p, q}|\xi|_{p+q}, \quad \xi \in E
$$

Notice that, because of assumption (2.3), $J$ commutes with $A$ therefore it induces a conjugation on all the Hilbert spaces $E_{p}$ with $p>0$ and can be extended to a conjugation on all $E_{p}$ with $p<0$. All these extensions will still be denoted by $J$. Thus it is a conjugation on all Hilbert spaces $\left(E_{p},(\cdot, \cdot)_{p}\right)(p \in \mathbb{R})$. In particular it is a continuous conjugation on $E$ and $E^{*}$ in the sense of topological vector spaces (antilinear and $J^{2}=\mathrm{id}$ ).

The fixed point subspaces of $J$ in $E$ (resp. $E^{*}$ ) will be denoted by $E_{\mathbb{R}}$ (resp. $\left.E_{\mathbb{R}}^{*}\right)$. They define the real triple:

$$
E_{\mathbb{R}} \subset H_{\mathbb{R}} \subset E_{\mathbb{R}}^{*}
$$

By constructing the space $E^{(0)}$ defined by (2.8), it is contained in $E$ and the restrictions on $H \times E$ of the natural duality $\left\langle E^{*}, E\right\rangle$ and of the $\mathbb{C}$-bilinear form $(2.1)$ coincide. When no confusion is possible, the natural duality $\left\langle E^{*}, E\right\rangle$ will also be denoted $\langle\cdot, \cdot\rangle$.

Lemma 2.3. For any $\alpha \in \mathbb{R}_{+}$, the operator $A^{\alpha}$ maps the space $E$ onto itself and its restriction on $E$ induces a topological vector space automorphism of $E$ with the projective limit topology. For $\alpha \leq 0, A^{\alpha}$ is a contraction with respect to any p-norm.

Proof. The elements of $E$ are those elements of $H$ that are in the domain of $A^{p}$ for each $p \in \mathbb{R}$. Therefore, for each $\alpha \in \mathbb{R}$, both $A^{\alpha}$ and $A^{-\alpha}$ are everywhere defined 
on $E$. $A^{\alpha}$ is surjective because, for each $\xi \in E, A^{\alpha}\left(A^{-\alpha} \xi\right)=\xi$. From the explicit form (2.5) of $A$ it follows that $A^{\alpha}$ is injective on $E$ and that its inverse is given by

$$
A^{-\alpha} \xi=\sum_{k=1}^{\infty} \ell_{k}^{-\alpha} \alpha_{k} e_{k} ; \quad \xi=\sum_{k=1}^{\infty} \alpha_{k} e_{k} \in E
$$

from this, the bijectivity of $A^{p}$ on $E$ follows. Thus $A^{\alpha}$ is a vector space automorphism of $E$ for any $\alpha \in \mathbb{R}$. Moreover, by inspection of (2.13), it follows that, if $\alpha \geq 0$, the $A^{-\alpha}$ is everywhere defined on $H$ and and $0<A^{-\alpha}<1$. To prove that it is also a topological automorphism in the projective limit topology on $E$ we prove continuity of $A^{\alpha}$ for each $\alpha \in \mathbb{R}$. This follows from the fact that for any $p \in \mathbb{R}$ and any $\xi \in E, \xi$ is in the domain of $A^{\alpha}$ and one has

$$
\left|A^{\alpha} \xi\right|_{p}^{2}=\left|A^{p+\alpha} \xi\right|_{0}^{2}=|\xi|_{p+\alpha}^{2} \leq|\xi|_{p+(\alpha+\beta)}^{2} ; \quad \forall \beta \geq 0,
$$

where the last inequality is due to the fact that the $p$-norms are increasing in $p$. Thus if $\alpha>0$ then for any $\beta \geq \alpha$ one has

$$
\left|A^{\alpha} \xi\right|_{p}^{2}=|\xi|_{p+\alpha}^{2} \leq|\xi|_{p+\beta}^{2} ; \quad \forall \xi \in E
$$

Hence $A^{\alpha}$ is continuous with respect to the projective topology. Finally if $\alpha \leq 0$, then $A^{\alpha}$ is a contraction for any $p$-norm because $0<A^{\alpha}<1$ on $H$ and $A^{\alpha}$ commutes with $A^{p}$ for any $p \in \mathbb{R}$.

Lemma 2.4. For any $\alpha \in \mathbb{R}$, the operator $A^{\alpha}$ maps the space $E^{*}$ onto itself and induces on it a topological vector space automorphism of $E^{*}$ with the inductive limit topology.

Proof. The operator $A$ is invertible on $H$ hence on all the spaces $E_{p} \subset H$ for $p>0$. If $p \leq 0$, then for any $\xi \in E_{p}$ one has

$$
|A \xi|_{p}^{2}=\left|A^{p+1} \xi\right|_{0}^{2}=|\xi|_{p+1}^{2} \geq|\xi|_{p}^{2}
$$

Therefore if $\xi \in E_{p}$ is such that $A \xi=0$, then also $|\xi|_{p}=0$ i.e., $\xi=0$. Therefore $A$ is injective on $E^{*}$. Since $A^{-1}$ is a contraction of the space $E_{p}$ into itself for all $p$-norms, then for any $\xi \in E^{*}$ one has $A\left(A^{-1} \xi\right)=\xi$ hence $A$ is surjective and is a vector space automorphism of $E^{*}$. Therefore the same is true for all its powers.

To prove that it is also a topological automorphism in the inductive limit topology on $E^{*}$ we prove continuity of $A^{\alpha}$ for each $\alpha \in \mathbb{R}$. We already know that, for $\alpha \leq 0, A^{\alpha}$ is a contraction of the space $E_{p}$ for all $p$-norms. Therefore we only have to consider the case $\alpha>0$. In this case one has, for any $p \geq \beta \geq \alpha$

$$
\left|A^{\alpha} \xi\right|_{-p}^{2}=\left|A^{\alpha-p} \xi\right|_{0}^{2}=|\xi|_{-p+\alpha}^{2} \leq|\xi|_{-(p-\beta)}^{2}
$$

and this proves the continuity of $A^{\alpha}$. 


\subsection{Second quantization}

For each $p \in \mathbb{R}$, let $\Gamma\left(E_{p}\right)$ be the (Boson) Fock space over the Hilbert space $E_{p}$ i.e.,

$$
\Gamma\left(E_{p}\right)=\left\{\phi=\left(f_{n}\right)_{n=0}^{\infty}: f_{n} \in E_{p}^{\widehat{\otimes} n},\|\phi\|_{p}^{2}=\sum_{n=0}^{\infty} n !\left|f_{n}\right|_{p}^{2}<\infty\right\}
$$

where $\widehat{\otimes} n$ is the $n$-fold symmetric tensor product. Then, identifying $\Gamma(H)$ with its dual space, we have the chain of Fock spaces:

$$
\cdots \subset \Gamma\left(E_{p}\right) \subset \Gamma\left(E_{0}\right)=\Gamma(H) \subset \Gamma\left(E_{-p}\right) \subset \cdots
$$

and a new triple

$$
(E)=\underset{p \rightarrow \infty}{\operatorname{proj}} \lim \Gamma\left(E_{p}\right) \subset \Gamma(H) \subset(E)^{*}=\operatorname{ind}_{p \rightarrow \infty} \lim _{p \rightarrow \infty} \Gamma\left(E_{-p}\right),
$$

where both projective and inductive limits are meant in the sense of topological vector spaces. The canonical $\mathbb{C}$-bilinear form on $(E)^{*} \times(E)$, denoted by $\langle\langle\cdot, \cdot\rangle\rangle$, has the form:

$$
\langle\langle\Phi, \phi\rangle\rangle=\sum_{n=0}^{\infty} n !\left\langle F_{n}, f_{n}\right\rangle ; \quad \Phi=\left(F_{n}\right) \in(E)^{*} ; \quad \phi=\left(f_{n}\right) \in(E),
$$

where, for each $n \in \mathbb{N},\left\langle F_{n}, f_{n}\right\rangle_{n}$ denotes the natural extension to $E^{* \otimes \hat{\otimes} n} \times E^{\hat{\otimes} n}$ of the the canonical $\mathbb{C}$-bilinear form on $E^{*} \times E$ and the suffix $n$ is omitted when no confusion is possible by writing simply an $\left\langle F_{n}, f_{n}\right\rangle$. In the notation (2.15), the exponential vector associated with $\xi \in H$ is defined by

$$
\phi_{\xi}:=\left(1, \xi, \frac{\xi^{\otimes 2}}{2 !}, \ldots, \frac{\xi^{\otimes n}}{n !}, \ldots\right),
$$

which can be extended to the vector $\xi \in E^{*}$. If $\xi, \eta \in E$, then for any $p \geq 0$, one has

$$
\left\langle\left\langle\phi_{\xi}, \phi_{\eta}\right\rangle\right\rangle=\exp \langle\xi, \eta\rangle
$$

therefore $\phi_{\xi} \in(E)$ for any $\xi \in E$. This implies that, for any element $\Phi \in(E)^{*}$, the map

$$
S \Phi(\cdot): E \ni \xi \mapsto S \Phi(\xi):=\left\langle\left\langle\Phi, \phi_{\xi}\right\rangle\right\rangle \in \mathbb{C}
$$

called the $S$-transform of the function $\Phi$, is well defined. A complex-valued function $F$ on $E$ is called a $U$-functional if $F$ is Gâteaux entire and there exist constants $C, K \geq 0$ and $p \geq 0$ such that

$$
|F(\xi)| \leq C \exp \left(K|\xi|_{p}^{2}\right), \quad \xi \in E .
$$

They are characterized by the following result.

Theorem 2.1. ${ }^{25}$ A complex-valued function $F$ on $E$ is the $S$-transform of an element $\Phi \in(E)^{*}$ if and only if $F$ is a $U$-functional. In this case $\Phi \in(E)^{*}$ is uniquely determined. 
Remark 2.2. The uniqueness result in Theorem 2.5 implies the invertibility of the $S$-transform from $(E)^{*}$ into $U$-functionals: a result frequently used in the following.

Remark 2.3. The continuity of the map $\xi \in E_{\mathbb{R}} \mapsto \exp (-\langle\xi, \xi\rangle / 2)$ and the Bochner-Minlos Theorem implies the existence of a probability measure $\mu$ on $E_{\mathbb{R}}^{*}$ such that

$$
\int_{E_{\mathbb{R}}^{*}} e^{i\langle x, \xi\rangle} d \mu(x)=e^{-\frac{1}{2}\langle\xi, \xi\rangle}, \quad \xi \in E_{\mathbb{R}} \cdot
$$

The Wiener-Itô-Segal isomorphism between $\Gamma(H)$ and $L^{2}\left(E^{*}, \mu\right)$ is the unitary isomorphism uniquely determined by the correspondence:

$$
\phi_{\xi}=\left(1, \xi, \frac{\xi^{\otimes 2}}{2 !}, \ldots, \frac{\xi^{\otimes n}}{n !}, \ldots\right) \leftrightarrow \phi_{\xi}(x)=e^{\langle x, \xi\rangle-\langle\xi, \xi\rangle / 2} ; \quad \xi \in E .
$$

We extend the identification of $\Gamma(H)$ with $L^{2}\left(E^{*}, \mu\right)$, provided by the Wiener-ItôSegal isomorphism, to the space $(E)$ of test functionals and to the space $(E)^{*}$ of white noise distributions (also called generalized white noise functionals), keeping the same notation for both when no confusion is possible. This gives the triple

$$
(E) \subset L^{2}\left(E^{*}, \mu\right) \subset(E)^{*},
$$

which is called the Hida-Kubo-Takenaka space. ${ }^{17}$

For any two topological vector spaces $\mathfrak{X}$ and $\mathfrak{Y}$, we denote

$$
\mathcal{L}(\mathfrak{X}, \mathfrak{Y}):=\text { the space of all continuous linear operators from } \mathfrak{X} \text { into } \mathfrak{Y} \text {. }
$$

With this notation the second quantization $\Gamma(T)$ of an operator $T \in \mathcal{L}\left(E, E^{*}\right)$ is defined on the exponential vectors by

$$
\Gamma(T) \phi_{\xi}=\phi_{T \xi} ; \quad \xi \in E .
$$

It is known (see Ref. 24) that

$$
\Gamma(T) \in \begin{cases}\mathcal{L}\left((E)^{*},(E)^{*}\right), & \text { if } T \in \mathcal{L}\left(E^{*}, E^{*}\right), \\ \mathcal{L}((E),(E)), & \text { if } T \in \mathcal{L}(E, E) .\end{cases}
$$

Theorem 2.2. (i) For any $\alpha \in \mathbb{R}$, the operator $\Gamma\left(A^{\alpha}\right)$ maps the space $(E)$ onto itself and its restriction on $(E)$ induces a topological vector space automorphism of $(E)$ with the projective limit topology. For $\alpha \leq 0, \Gamma\left(A^{\alpha}\right)$ is a contraction with respect to any p-norm.

(ii) For any $\alpha \in \mathbb{R}$, the operator $\Gamma\left(A^{\alpha}\right)$ maps the space $(E)^{*}$ onto itself and induces on it a topological vector space automorphism of $(E)^{*}$ with the projective limit topology.

Proof. The statements follow from Lemmata (2.3), (2.4) and the properties (2.20) combined with the functorial properties of $\Gamma$, in particular

$$
\begin{gathered}
\Gamma\left(\left(A^{\alpha}\right)^{*}\right)=\left(\Gamma\left(A^{\alpha}\right)\right)^{*} ; \\
\Gamma\left(\left(A^{\alpha}\right)^{-1}\right)=\left(\Gamma\left(A^{\alpha}\right)\right)^{-1} .
\end{gathered}
$$




\section{Derivatives of White Noise}

\subsection{The choice of the space $H \subset L^{2}([0,1])$}

In the following, we fix $H$ to be the orthogonal complement of the constant functions in $L^{2}([0,1])$ with the conjugation operator $J \xi=\bar{\xi}$ defined by pointwise complex conjugation. For each $t \in \mathbb{R}$, let $U_{t}$ be the linear operator on $L^{2}([0,1])$ defined as the right translation by $t$ modulo 1 :

$$
\left(U_{t} f\right)(s):=f(s+t(\bmod 1)) ; \quad f \in L^{2}(0,1), s \in(0,1) .
$$

The set of functions $e \equiv\left\{e_{n}\right\}_{n=1}^{\infty}$

$$
e_{2 k-1}(t):=e^{2 \pi i k t} ; \quad e_{2 k}(t):=e^{-2 \pi i k t} ; \quad k \in \mathbb{N}^{*}:=\mathbb{N} \backslash\{0\}, \quad t \in \mathbb{R}
$$

is an orthonormal basis of $H$ and on it $U_{t}$ acts as

$$
\left(U_{t} e^{ \pm i 2 \pi n(\cdot)}\right)(s):=e^{ \pm i 2 \pi n(t+s)}=e^{ \pm i 2 \pi n t}\left(e^{ \pm i 2 \pi n(\cdot)}\right)(s) .
$$

In particular the elements of the orthonormal basis (3.2) of $H$ are eigenvectors of the operator $U_{t}$ corresponding to the eigenvalues $e^{i 2 \pi n t}(n \in \mathbb{Z})$. From this, one easily checks that $\left(U_{t}\right)_{t \in \mathbb{R}}$ is a strongly continuous 1-parameter unitary group on $L^{2}([0,1])$.

The constant function $1 \in L^{2}([0,1])$ is invariant under the action of $\left(U_{t}\right)$, therefore the same is true for its orthogonal complement i.e., $H$. Thus the restriction of $\left(U_{t}\right)$ on $H$ gives a 1-parameter unitary group still denoted $\left(U_{t}\right)$. By Stone's theorem, the generator of $\left(U_{t}\right)$ is the form $i A$ where $A$ is a self-adjoint operator. From (3.1) it follows that, on the subspace of $H$ made of (classes of) differentiable functions, the operator $A$ coincides with the momentum operator

$$
p=\frac{1}{i} \partial
$$

where $\partial$ is the partial derivative in the variable $t$. We will use the same symbol $\partial$ to denote the derivation operator and its restriction on $H$. The self-adjointness of $A$ implies that

$$
p^{*}=-\frac{1}{i} \partial^{*}=p=\frac{1}{i} \partial
$$

i.e., on the domain of $p$ :

$$
\partial^{*}=-\partial,
$$

where all adjoints are meant on $H$. This can also be checked directly because the invariance of $H$ under $p$ implies that, if $f \in H$ is in the domain of $p$, then $f(0)=f(1)$.

\subsection{The standard triple associated to $H$}

We have seen that the elements of the orthonormal basis (3.2) of $H$ are eigenvectors of the operator $\partial$ or equivalently of $p$ :

$$
p e_{2 k-1}(t)=2 \pi k e_{2 k-1}(t) ; \quad p e_{2 k}(t)=-2 \pi k e_{2 k}(t) ; \quad k \in \mathbb{N}^{*} .
$$


Therefore the operator $p$ on $H$ satisfies conditions (2.2), (2.3), (2.4) and in this case the $\ell_{n}\left(\in \mathbb{N}^{*}\right)$ are given by

$$
\ell_{n}:=(-1)^{n+1} 2[(n+1) / 2] \pi .
$$

By the spectral theorem, for any $\alpha \in \mathbb{R}_{+}, p^{\alpha}$ is well defined taking the principal value on the negative part of the spectrum and its action on the linear span of the basis $e$, defining $E^{(0)}$ (see $(2.8)$ ), is explicitly given by

$$
p^{\alpha} e_{n}=\ell_{n}^{\alpha} e_{n} ; \quad n \in \mathbb{N}^{*} .
$$

Therefore all the results obtained in Sec. 2 are applicable to the operator $p$. In particular Lemma 2.3 becomes in this case:

Lemma 3.1. For any $\alpha \in \mathbb{R}_{+}$, the operator $p^{\alpha}$ is a topological vector space automorphism of the space $E$ with the projective limit topology. For $\alpha \leq 0, p^{\alpha}$ is a contraction with respect to any p-norm.

Similarly Lemma 2.4 becomes:

Lemma 3.2. For any $\alpha \in \mathbb{R}$, the operator $p^{\alpha}$ is a topological vector space automorphism of the space $E^{*}$ with the inductive limit topology.

Remark 3.1. Since the operator $p$ differs from $\partial$ only by multiplication for the imaginary unit, the conclusions of both Lemmas 3.1 and 3.2 continue to take place, on the same domains, also for $\partial$ and for $\partial^{*}=-\partial$.

Finally Theorem 2.2 becomes in this case:

Theorem 3.1. (i) For any $\alpha \in \mathbb{R}$, the operator $\Gamma\left(p^{\alpha}\right)$ maps the space $(E)$ onto itself and its restriction on $(E)$ induces a topological vector space automorphism of (E) with the projective limit topology. For $\alpha \leq 0, \Gamma\left(p^{\alpha}\right)$ is a contraction with respect to any $p$-norm.

(ii) For any $\alpha \in \mathbb{R}$, the operator $\Gamma\left(p^{\alpha}\right)$ maps the space $(E)^{*}$ onto itself and induces on it a topological vector space automorphism of $(E)^{*}$ with the projective limit topology.

(iii) Properties (i) and (ii) continue to hold if the operator $\Gamma\left(p^{\alpha}\right)$ is replaced by $\Gamma\left(\partial_{\alpha}\right)$.

Proof. We only have to prove (iii) because (i) and (ii) are particular cases of Theorem 2.2. Notice that the functorial properties of $\Gamma$ which imply that

$$
\Gamma\left(p^{\alpha}\right)=\Gamma\left(i^{-\alpha}\right) \Gamma\left(\partial_{\alpha}\right),
$$

where $i^{-\alpha}$ is defined by taking (when necessary) the principal part of the logarithm. Equivalently

$$
\Gamma\left(\partial_{\alpha}\right)=\Gamma\left(i^{\alpha}\right) \Gamma\left(p^{\alpha}\right)=\Gamma\left((i p)^{\alpha}\right) .
$$

The thesis then follows because $\Gamma\left(i^{\alpha}\right)$ is unitary on all the $\Gamma\left(E_{p}\right)$ spaces and a topological automorphism on both $(E)$ and $(E)^{*}$ and a composition of topological automorphisms is a topological automorphism. 


\section{Higher Order Cesàro Mean}

Accardi and Smolyanov in Ref. 5 proved the following generalization, to higher order means, of Cesàro's theorem on arithmetic means.

Theorem 4.1. Let $p>0$ and let $a=\left(a_{n}\right)_{n=1}^{\infty}$ be a sequence in $\mathbb{C}^{\infty}$ such that the limit

$$
\lim _{N \rightarrow \infty} \frac{1}{N^{p}} \sum_{n=1}^{N} a_{n}=: A_{p}(a)
$$

exists. Then

$$
\lim _{N \rightarrow \infty} \frac{1}{N^{p+1}} \sum_{n=1}^{N} n a_{n}=\frac{p}{p+1} A_{p}(a) .
$$

For the purposes of the present paper, the following generalization of Theorem 4.1 is needed.

Theorem 4.2. Let the sequence $a=\left(a_{n}\right)_{n=1}^{\infty} \in \mathbb{C}^{\infty}$ be such that, for some $p>0$ the limit

$$
\lim _{N \rightarrow \infty} \frac{1}{N^{p}} \sum_{n=1}^{N} a_{n}=: A_{p}(a)
$$

exists. Then for each $\alpha \in \mathbb{R}_{+}$, one has

$$
\lim _{N \rightarrow \infty} \frac{1}{N^{p+\alpha}} \sum_{n=1}^{N} n^{\alpha} a_{n}=\frac{p}{p+\alpha} A_{p}(a)
$$

in the sense that the limit on the left-hand side exists and the equality holds.

Proof. The Abel identity implies that, for arbitrary sequences $\left(a_{n}\right),\left(b_{n}\right)$ in $\mathbb{C}^{\infty}$ and any natural number $N \geq 2$, one has:

$$
\sum_{k \leq N} a_{k} b_{k}=\left(\sum_{k \leq N} a_{k}\right) b_{N}-\sum_{k \leq N-1}\left(b_{k+1}-b_{k}\right)\left(\sum_{r \leq k} a_{r}\right) .
$$

Using this with $b_{k}=k^{\alpha}$ we see that

$$
\begin{aligned}
\frac{1}{N^{p+\alpha}} \sum_{n=1}^{N} n^{\alpha} a_{n} & =\frac{1}{N^{p+\alpha}}\left[\left(\sum_{n=1}^{N} a_{n}\right) N^{\alpha}-\sum_{n=1}^{N-1}\left\{(n+1)^{\alpha}-n^{\alpha}\right\} \sum_{k=1}^{n} a_{k}\right] \\
& =\frac{1}{N^{p}} \sum_{n=1}^{N} a_{n}-\frac{\alpha}{N^{p+\alpha}} \sum_{n=1}^{N-1} \int_{n}^{n+1} x^{\alpha-1} d x \sum_{k=1}^{n} a_{k} \\
& =\frac{1}{N^{p}} \sum_{n=1}^{N} a_{n}-\frac{\alpha}{N^{p+\alpha}} \sum_{n=1}^{N-1} \int_{n}^{n+1} x^{p+\alpha-1}\left(\frac{1}{x^{p}} \sum_{k=1}^{n} a_{k}\right) d x .
\end{aligned}
$$


Therefore we have

$$
\frac{1}{N^{p+\alpha}} \sum_{n=1}^{N} n^{\alpha} a_{n}=I_{1}(p, \alpha, N)+I_{2}(p, \alpha, N)+I_{3}(p, \alpha, N),
$$

where

$$
\begin{aligned}
& I_{1}(p, \alpha, N)=\frac{1}{N^{p}} \sum_{n=1}^{N} a_{n} \\
& I_{2}(p, \alpha, N)=-\frac{\alpha}{N^{p+\alpha}} \sum_{n=1}^{N-1} \int_{n}^{n+1} x^{p+\alpha-1} d x A_{p}(a), \\
& I_{3}(p, \alpha, N)=-\frac{\alpha}{N^{p+\alpha}} \sum_{n=1}^{N-1} \int_{n}^{n+1} x^{p+\alpha-1}\left(\frac{1}{x^{p}} \sum_{k=1}^{n} a_{k}-A_{p}(a)\right) d x .
\end{aligned}
$$

Since

$$
I_{2}(p, \alpha, N)=-\frac{\alpha}{p+\alpha} \frac{N^{p+\alpha}-1}{N^{p+\alpha}} A_{p}(a),
$$

we have

$$
\lim _{N \rightarrow \infty} I_{2}(p, \alpha, N)=\frac{\alpha}{p+\alpha} A_{p}(a) .
$$

Since

$$
\left|I_{3}(p, \alpha, N)\right| \leq \frac{\alpha}{N^{p+\alpha}} \sum_{n=1}^{N-1} \int_{n}^{n+1} x^{p+\alpha-1}\left|\frac{1}{x^{p}} \sum_{k=1}^{n} a_{k}-A_{p}(a)\right| d x
$$

with

$$
\lim _{n \rightarrow \infty} \max _{n \leq x \leq n+1}\left|\frac{1}{x^{p}} \sum_{k=1}^{n} a_{k}-A_{p}(a)\right|=0
$$

by assumption, for any $\epsilon>0$ one can find $N_{\epsilon} \in \mathbb{N}$ and a constant $C>0$ such that

$$
\begin{aligned}
\left|I_{3}(p, \alpha, N)\right| & \leq C \frac{\alpha}{N^{p+\alpha}} \sum_{n=1}^{N_{\epsilon}-1} \int_{n}^{n+1} x^{p+\alpha-1} d x+\epsilon \frac{\alpha}{N^{p+\alpha}} \sum_{n=N_{\epsilon}}^{N-1} \int_{n}^{n+1} x^{p+\alpha-1} d x \\
& =C \frac{\alpha}{p+\alpha} \frac{N_{\epsilon}^{p+\alpha}-1}{N^{p+\alpha}}+\epsilon \frac{\alpha}{p+\alpha} \frac{N^{p+\alpha}-N_{\epsilon}^{p+\alpha}}{N^{p+\alpha}} \\
& \leq C \frac{\alpha}{p+\alpha} \frac{N_{\epsilon}^{p+\alpha}-1}{N^{p+\alpha}}+\frac{\epsilon \alpha}{p+\alpha} .
\end{aligned}
$$

For $N \rightarrow \infty$ this remains $\leq 2 \epsilon$. Since $\epsilon$ is arbitrary, we conclude that

$$
\lim _{N \rightarrow \infty} I_{3}(p, \alpha, N)=0
$$


Therefore

$$
\begin{aligned}
\lim _{N \rightarrow \infty} \frac{1}{N^{p+\alpha}} \sum_{n=1}^{N} n^{\alpha} a_{n} & =\lim _{N \rightarrow \infty} \sum_{i=1}^{3} I_{i}(p, \alpha, N) \\
& =A_{p}(a)-\frac{\alpha}{p+\alpha} A_{p}(a)=\frac{p}{p+\alpha} A_{p}(a),
\end{aligned}
$$

where the limit on the left-hand side exists because the limit on the right-hand side exists by assumption. This proves the statement.

By taking $b_{k}=2[(k+1) / 2]^{\alpha}, k=1,2, \ldots$ in the proof of Theorem 4.2, we have the following:

Corollary 4.1. Let $a=\left(a_{n}\right)_{n=1}^{\infty} \in \mathbb{C}^{\infty}$ be such that, for some $p>0$ the limit

$$
\lim _{N \rightarrow \infty} \frac{1}{N^{p}} \sum_{n=1}^{N} a_{n}=: A_{p}(a)
$$

exists. Then for each $\alpha \in \mathbb{R}_{+}$, one has

$$
\lim _{N \rightarrow \infty} \frac{1}{N^{p+\alpha}} \sum_{n=1}^{N}(2[(n+1) / 2])^{\alpha} a_{n}=\frac{p}{p+\alpha} A_{p}(a) .
$$

The following theorem gives the converse of Theorem 4.2.

Theorem 4.3. Let $p>0, \alpha \geq 0$ and let $a=\left(a_{n}\right)_{n=1}^{\infty}$ be a sequence in $\mathbb{C}^{\infty}$ such that the limit

$$
\lim _{N \rightarrow \infty} \frac{1}{N^{p+\alpha}} \sum_{n=1}^{N} a_{n}=: A_{p+\alpha}(a)
$$

exists. Then

$$
\lim _{N \rightarrow \infty} \frac{1}{N^{p}} \sum_{n=1}^{N} n^{-\alpha} a_{n}=\frac{p+\alpha}{p} A_{p+\alpha}(a) .
$$

Proof. If $\alpha=0$, then the statement is obvious. By applying (4.2) and the mean value theorem, we obtain that for any $N \geq 2$ and $1 \leq n \leq N$, there exists $x_{n} \in$ $[n, n+1]$ such that

$$
\begin{aligned}
\frac{1}{N^{p}} \sum_{n=1}^{N} n^{-\alpha} a_{n} & =\frac{1}{N^{p}}\left(\frac{1}{N^{\alpha}} \sum_{n=1}^{N} a_{n}-\sum_{n=1}^{N-1}\left((n+1)^{-\alpha}-n^{-\alpha}\right)\left(\sum_{k=1}^{n} a_{k}\right)\right) \\
& =\frac{1}{N^{p+\alpha}} \sum_{n=1}^{N} a_{n}+\alpha \frac{1}{N^{p}} \sum_{n=1}^{N-1}\left(\int_{n}^{n+1} x^{-(\alpha+1)} d x\right)\left(\sum_{k=1}^{n} a_{k}\right) \\
& =\frac{1}{N^{p+\alpha}} \sum_{n=1}^{N} a_{n}+\alpha \frac{1}{N^{p}} \sum_{n=1}^{N-1} n^{p+\alpha} x_{n}^{-(\alpha+1)}\left(\frac{1}{n^{p+\alpha}} \sum_{k=1}^{n} a_{k}\right) .
\end{aligned}
$$


Therefore, we have

$$
\frac{1}{N^{p}} \sum_{n=1}^{N} n^{-\alpha} a_{n}=I_{1}(p, \alpha, N)+I_{2}(p, \alpha, N)+I_{3}(p, \alpha, N),
$$

where

$$
\begin{aligned}
& I_{1}(p, \alpha, N)=\frac{1}{N^{p+\alpha}} \sum_{n=1}^{N} a_{n}, \\
& I_{2}(p, \alpha, N)=\alpha \frac{1}{N^{p}} \sum_{n=1}^{N-1} n^{p+\alpha} x_{n}^{-(\alpha+1)} A_{p+\alpha}(a), \\
& I_{3}(p, \alpha, N)=\alpha \frac{1}{N^{p}} \sum_{n=1}^{N-1} n^{p+\alpha} x_{n}^{-(\alpha+1)}\left(\frac{1}{n^{p+\alpha}} \sum_{k=1}^{n} a_{k}-A_{p+\alpha}(a)\right) .
\end{aligned}
$$

Then by assumption, $\lim _{N \rightarrow \infty} I_{1}(p, \alpha, N)=A_{p+\alpha}(a)$. On the other hand, we have

$$
\begin{aligned}
I_{2}(p, \alpha, N)= & \alpha \sum_{n=1}^{N-1}\left(\frac{n}{N}\right)^{p-1}\left(\frac{1}{N}\right)\left(\frac{n}{x_{n}}\right)^{\alpha+1} A_{p+\alpha}(a) \\
= & \alpha \sum_{n=1}^{N-1}\left(\frac{n}{N}\right)^{p-1}\left(\frac{1}{N}\right) A_{p+\alpha}(a) \\
& +\alpha \frac{1}{N} \sum_{n=1}^{N-1}\left(\frac{n}{N}\right)^{p-1}\left[\left(\frac{n}{x_{n}}\right)^{\alpha+1}-1\right] A_{p+\alpha}(a),
\end{aligned}
$$

of which the first and second terms converge to $\alpha A_{p+\alpha}(a) / p$ and 0 respectively as $N \rightarrow \infty$. Therefore, $\lim _{N \rightarrow \infty} I_{2}(p, \alpha, N)=\alpha A_{p+\alpha}(a) / p$. By similar arguments, we can easily see that $\lim _{N \rightarrow \infty} I_{3}(p, \alpha, N)=0$. Hence by (4.6), we see that

$$
\lim _{N \rightarrow \infty} \frac{1}{N^{p}} \sum_{n=1}^{N} n^{-\alpha} a_{n}=\lim _{N \rightarrow \infty} \sum_{i=1}^{3} I_{i}(p, \alpha, N)=A_{p+\alpha}(a)+\frac{\alpha}{p} A_{p+\alpha}(a),
$$

which proves (4.5).

\section{Exotic Laplacians and Their Mutual Relationships}

A function $F: E \rightarrow \mathbb{C}$ is said to be of class $C^{2}$ if it is twice (continuously) Fréchet differentiable, i.e., there exist two continuous maps

$$
\xi \mapsto F^{\prime}(\xi) \in E^{*}, \quad \xi \mapsto F^{\prime \prime}(\xi) \in \mathcal{L}\left(E, E^{*}\right), \quad \xi \in E
$$

such that for any $\eta \in E$

$$
F(\xi+\eta)=F(\xi)+\left\langle F^{\prime}(\xi), \eta\right\rangle+\frac{1}{2}\left\langle F^{\prime \prime}(\xi) \eta, \eta\right\rangle+\epsilon(\eta),
$$


where the error term $\epsilon(\eta)$ satisfies

$$
\lim _{t \rightarrow 0} \frac{\epsilon(t \eta)}{t^{2}}=0, \quad \eta \in E .
$$

We denote $\widetilde{D}_{\eta}$ the Gateaux differentiation in the direction $\eta$, i.e.,

$$
\widetilde{D}_{\eta} F(\xi)=\lim _{t \rightarrow 0} \frac{1}{t}[F(\xi+t \eta)-F(\xi)]=\left.\frac{d}{d t} F(\xi+t \eta)\right|_{t=0} .
$$

It is known (see Ref. 14) that, under general regularity conditions on $F$, one has

$$
\widetilde{D}_{\eta} F(\xi)=\left\langle F^{\prime}(\xi), \eta\right\rangle
$$

in the sense that, the existence of either side of the identity implies the existence of the other one and the equality. The kernel theorem identifies $\mathcal{L}\left(E, E^{*}\right)$ with $E^{*} \otimes E^{*}$ (see Ref. 10 or 24). Using this identification we will use indifferently the notations:

$$
\left\langle F^{\prime \prime}(\xi) \eta, \eta\right\rangle=\left\langle F^{\prime \prime}(\xi), \eta \otimes \eta\right\rangle=F^{\prime \prime}(\xi)(\eta, \eta)=\widetilde{D}_{\eta}^{2} F(\xi) .
$$

For $\alpha \in \mathbb{R}_{+}$, let $\operatorname{Dom}\left(\Delta_{c, \alpha}\right)$ denote the set of all $\Phi \in(E)^{*}$ such that the limit

$$
\widetilde{\Delta}_{c, \alpha} S \Phi(\xi):=\lim _{N \rightarrow \infty} \frac{1}{N^{\alpha}} \sum_{k=1}^{N}\left\langle(S \Phi)^{\prime \prime}(\xi), e_{k} \otimes e_{k}\right\rangle
$$

exists for each $\xi \in E$ and is a $U$-functional. The exotic Laplacian of order $\alpha \in \mathbb{R}_{+}$, denoted by $\Delta_{c, \alpha}$, is defined on $\operatorname{Dom}\left(\Delta_{c, \alpha}\right)$ by

$$
\Delta_{c, \alpha} \Phi:=S^{-1}\left(\widetilde{\Delta}_{c, \alpha} S \Phi\right), \quad \Phi \in \operatorname{Dom}\left(\Delta_{c, \alpha}\right) .
$$

Since the $S$-transform is the white noise analogue of the Fourier transform, one can say that $\widetilde{\Delta}_{c, \alpha}$ is the exotic Laplacian of order $\alpha$ in momentum representation. The operator $\Delta_{c, 1}$ is called the Lévy Laplacian and is denoted by $\Delta_{L}$.

Using the bijectivity of $\Gamma\left(\partial_{\alpha}^{*}\right)$, we introduce the Lévy Laplacian $\Delta_{L, \alpha}$ of order $\alpha$, defined on the domain $\Gamma\left(\partial_{\alpha}^{*}\right)\left(\operatorname{Dom}\left(\Delta_{L}\right)\right)$ of the derivatives of order $\alpha$ of white noise functionals, by

$$
\Delta_{L, \alpha} \Phi=\Gamma\left(\partial_{\alpha}^{*}\right) \Delta_{L} \Gamma\left(\partial_{\alpha}^{*}\right)^{-1} \Phi ; \quad \Phi \in \Gamma\left(\partial_{\alpha}^{*}\right)\left(\operatorname{Dom}\left(\Delta_{L}\right)\right) \subseteq(E)^{*} .
$$

Theorem 5.1. Let $K \in \mathbb{R}_{+}$and $\Phi \in \operatorname{Dom}\left(\Delta_{c, 2 K+1}\right)$. Then for any $\alpha \in \mathbb{R}_{+}$,

$$
\Gamma\left(p^{\alpha}\right) \Phi \in \operatorname{Dom}\left(\Delta_{c, 2(\alpha+K)+1}\right)
$$

and the identity:

$$
\Delta_{c, 2(\alpha+K)+1} \Gamma\left(p^{\alpha}\right) \Phi=\frac{\pi^{2 \alpha}(2 K+1)}{2(\alpha+K)+1} \Gamma\left(p^{\alpha}\right) \Delta_{c, 2 K+1} \Phi
$$

holds. 
Proof. Let $\Phi$ be a functional in $\operatorname{Dom}\left(\Delta_{c, 2 K+1}\right)$. Then applying Theorem 4.2 , we obtain that for any $\xi \in E$

$$
\begin{aligned}
S\left(\Delta_{c, 2(\alpha+K)+1} \Gamma\left(p^{\alpha}\right) \Phi\right)(\xi) & =\lim _{N \rightarrow \infty} \frac{1}{N^{2(\alpha+K)+1}} \sum_{n=1}^{N} S \Phi^{\prime \prime}\left(\xi^{(\alpha)}\right)\left(p^{\alpha} e_{n}, p^{\alpha} e_{n}\right) \\
& =\lim _{N \rightarrow \infty} \frac{1}{N^{2(\alpha+K)+1}} \sum_{n=1}^{N} \ell_{n}^{2 \alpha} S \Phi^{\prime \prime}\left(\xi^{(\alpha)}\right)\left(e_{n}, e_{n}\right) \\
& =\frac{\pi^{2 \alpha}(2 K+1)}{2(\alpha+K)+1} \cdot \lim _{N \rightarrow \infty} \frac{1}{N^{2 K+1}} \sum_{n=1}^{N} S \Phi^{\prime \prime}\left(\xi^{(\alpha)}\right)\left(e_{n}, e_{n}\right) \\
& =\frac{\pi^{2 \alpha}(2 K+1)}{2(\alpha+K)+1} \cdot S\left[\Delta_{c, 2 K+1} \Phi\right]\left(\xi^{(\alpha)}\right) .
\end{aligned}
$$

The assertion follows because (see Theorem 3.3 of Ref. 7 )

$$
S\left[\Delta_{c, 2 K+1} \Phi\right]\left(\xi^{(\alpha)}\right)=S\left[\Gamma\left(p^{\alpha}\right) \Delta_{c, 2 K+1} \Phi\right](\xi) .
$$

Corollary 5.1. Let $\Phi \in \operatorname{Dom}\left(\Delta_{L}\right)$. Then for any $\alpha \in \mathbb{R}_{+}, \Gamma\left(p^{\alpha}\right) \Phi \in \operatorname{Dom}\left(\Delta_{c, 2 \alpha+1}\right)$ and the identity:

$$
\Delta_{c, 2 \alpha+1} \Gamma\left(p^{\alpha}\right) \Phi=\frac{\pi^{2 \alpha}}{2 \alpha+1} \Gamma\left(p^{\alpha}\right) \Delta_{L} \Phi=\frac{\pi^{2 \alpha}}{2 \alpha+1} \Delta_{L, \alpha} \Gamma\left(p^{\alpha}\right) \Phi
$$

holds.

Proof. The proof is immediate from Theorem 5.1 by taking $K=0$.

\section{Exotic Traces and Exotic Triples}

The Cesàro semi-norm of order $\alpha$ of $x \in E^{*}$ is defined by

$$
|x|_{c, \alpha}^{2}:=\lim _{N \rightarrow \infty} \frac{1}{N^{\alpha}} \sum_{n=1}^{N} \overline{\left\langle x, e_{n}\right\rangle}\left\langle x, e_{n}\right\rangle
$$

in the sense that, when the limit exists, the semi-norm is defined by the above limit.

Remark 6.1. Since $\bar{e}_{2 k}=e_{2 k-1}, \bar{e}_{2 k-1}=e_{2 k}, k=1,2,3, \ldots$, we have

$$
|x|_{c, \alpha}^{2}=\lim _{N \rightarrow \infty} \frac{1}{N^{\alpha}} \sum_{n=1}^{N} \overline{\left\langle x, \bar{e}_{n}\right\rangle}\left\langle x, \bar{e}_{n}\right\rangle
$$

for $x, y \in E^{*}$.

The Cesàro $\mathbb{C}$-bilinear form of order $\alpha$ between $x, y \in E^{*}$ is defined, in the same sense, by

$$
\langle x, y\rangle_{c, \alpha}:=\lim _{N \rightarrow \infty} \frac{1}{N^{\alpha}} \sum_{n=1}^{N}\left\langle x, e_{n}\right\rangle\left\langle y, e_{n}\right\rangle .
$$


For all $\alpha \geq 1$ and $\lambda \in \mathbb{R}$, define

$$
x_{\lambda, \alpha}:=\sum_{n=1}^{\infty} e^{2 \pi i \lambda[(n+1) / 2]} e_{n}^{((\alpha-1) / 2)}=\sum_{k=1}^{\infty} e^{2 \pi i \lambda k} e_{2 k}^{((\alpha-1) / 2)}+\sum_{k=1}^{\infty} e^{-2 \pi i \lambda k} e_{2 k-1}^{((\alpha-1) / 2)}
$$

based on the expansion of the $\frac{(\alpha-1)}{2}$ th derivative of delta function $\delta_{\lambda}$. By (3.7) we have the following.

Lemma 6.1. (cf. Ref. 6) For all $\alpha \geq 1$ and $\lambda \in \mathbb{R}, x_{\lambda, \alpha} \in E^{*}$.

Lemma 6.2. (cf. Ref. 6) For all $\alpha \geq 1$ and $\lambda, \mu \in \mathbb{R} \backslash \mathbb{Q}$, we have

$$
\left.\left\langle J\left(x_{\lambda, \alpha}\right), x_{\mu, \alpha}\right\rangle_{c, \alpha}=\frac{(2 \pi)^{\alpha-1}}{\alpha} \delta_{\lambda, \mu} \quad \text { (Kronecker's delta }\right)
$$

Let $\mathcal{C}$ be a countable set in $\mathbb{R} \backslash \mathbb{Q}$. Then, from Lemma 6.2 we know that, for each $\alpha \geq 1$, the set

$$
\left\{e_{\alpha, \lambda}:=x_{\lambda, \alpha} \sqrt{\alpha /(2 \pi)^{\alpha-1}}: \lambda \in \mathcal{C}\right\}
$$

is orthonormal for the scalar product $\langle J \cdot, \cdot\rangle_{c, \alpha}$. Therefore, the space

$$
H_{c, \alpha}^{\circ}:=\text { linear } \operatorname{span}\left\{e_{\alpha, \lambda}: \lambda \in \mathcal{C}\right\}
$$

is a pre-Hilbert space with the inner product

$$
\langle J \xi, \eta\rangle_{c, \alpha}=\lim _{N \rightarrow \infty} \frac{1}{N^{\alpha}} \sum_{n=1}^{N} \overline{\left\langle\xi, e_{n}\right\rangle}\left\langle\eta, e_{n}\right\rangle ; \quad \xi, \eta \in H_{c, \alpha}^{\circ} .
$$

Let $H_{c, \alpha}$ be the completion of the pre-Hilbert space $H_{c, \alpha}^{\circ}$ with respect to the norm induced by the inner product $\langle J \cdot, \cdot\rangle_{c, \alpha}$. In general, $H_{c, \alpha}$ will not be contained in $E^{*}$. Then $H_{c, \alpha}$ becomes an infinite dimensional separable Hilbert space whose inner product will still be denoted by $\langle J \cdot, \cdot\rangle_{c, \alpha}$ when no confusion is possible and, by construction, the set $\left\{e_{\alpha, \lambda}\right\}_{\lambda \in \mathcal{C}}$ is an orthonormal basis of $H_{c, \alpha}$. Relabeling the orthonormal basis $\left\{e_{\alpha, \lambda}\right\}_{\lambda \in \mathcal{C}}$ of $H_{c, \alpha}$ we use the notation $\left\{e_{\alpha, k}\right\}_{k=1}^{\infty}$.

For $\lambda \in[0,1]$ define $\delta_{\lambda} \in E^{*}$ by $\delta_{\lambda}(\xi)=\xi(\lambda), \xi \in E$. Take a countable set $\mathcal{C}$ in $[0,1] \backslash \mathbb{Q}$ and let

$$
\mathcal{E}:=\text { linear } \operatorname{span}\left\{\partial_{1 / 2}^{-1} \delta_{\lambda}: \lambda \in \mathcal{C}\right\}
$$

Then $\mathcal{E}$ is in $E^{*}$ and since

$$
\partial_{1 / 2}^{-1} \delta_{\lambda}=\sum_{k=1}^{\infty}\left\{(2 \pi i k)^{-1 / 2} e^{-2 \pi i \lambda k} e_{2 k}+(-2 \pi i k)^{-1 / 2} e^{2 \pi i \lambda k} e_{2 k-1}\right\},
$$

we have

$$
\lim _{n \rightarrow \infty} n\left|\left\langle\partial_{1 / 2}^{-1} \delta_{\lambda}, e_{n}\right\rangle\right|^{2}=\frac{1}{\pi} .
$$


For $\alpha \in \mathbb{R}$ and $N \in \mathbb{N}$ we define the pre-scalar product $\langle\cdot, \cdot\rangle_{\alpha}$ on $E^{*}$ by

$$
\begin{aligned}
\left\langle\partial_{\alpha / 2} \xi, \partial_{\alpha / 2} \eta\right\rangle_{\alpha, N} & :=\sum_{n=1}^{N} \overline{\left\langle\partial_{\alpha / 2} \xi, e_{n}\right\rangle}\left\langle\partial_{\alpha / 2} \eta, e_{n}\right\rangle, \\
\left\langle\partial_{\alpha / 2} \xi, \partial_{\alpha / 2} \eta\right\rangle_{\alpha} & :=\lim _{N \rightarrow \infty} \frac{1}{N^{\alpha}}\left\langle\partial_{\alpha / 2} \xi, \partial_{\alpha / 2} \eta\right\rangle_{\alpha, N}
\end{aligned}
$$

for $\xi$ and $\eta$ in $\mathcal{E}$. Then it coincides with the Cesàro pre-scalar product $\left\langle\partial_{\alpha / 2} \xi, \partial_{\alpha / 2} \eta\right\rangle_{c, \alpha}$. Let

$$
W_{\alpha}:=\left\{\partial_{\alpha / 2} \xi: \xi \in \mathcal{E}\right\}
$$

Then we have $W_{\alpha} \subset H_{c, \alpha}$ since

$$
\left\langle\partial_{\alpha / 2} \xi, \partial_{\alpha / 2} \eta\right\rangle_{\alpha}=\lim _{N \rightarrow \infty} \frac{1}{N^{\alpha}} \sum_{k=1}^{N}(2 \pi[(k+1) / 2])^{\alpha-1} \overline{\left\langle\xi, e_{k}\right\rangle}\left\langle\eta, e_{k}\right\rangle
$$

exists by Theorem 4.2 , and $e_{\alpha, \lambda}$ is given by the $\frac{(\alpha-1)}{2}$ th derivative of the delta function $\delta_{\lambda}$ for $\alpha \geq 1$ and $\lambda \in[0,1]$. Thus we have the following:

Theorem 6.1. For $\alpha \geq 1$ the completion of $W_{\alpha}$ with respect to $\langle\cdot, \cdot\rangle_{\alpha}$ coincides with $H_{c, \alpha}$.

The construction in Sec. 2 can be applied to the space $H_{c, \alpha}$ replacing the sequence $\left\{\ell_{k}\right\}$ by an arbitrary sequence $\left\{\ell_{c, k}\right\}$ satisfying

$$
1<\ell_{c, 1} \leq \ell_{c, 2} \leq \ell_{c, 3} \leq \cdots \text { and } \sum_{k=1}^{\infty} \ell_{c, k}^{-2}<\infty
$$

and introducing the densely defined selfadjoint operator on $\left(H_{c, \alpha},\left\{e_{\alpha, k}\right\}\right)$

$$
A_{\alpha}:=\sum_{k=1}^{\infty} \ell_{c, k} d_{k} e_{\alpha, k}, \quad \xi=\sum_{k=1}^{\infty} d_{k} e_{a, k} \in H_{c, a} .
$$

We also assume that for any $\alpha>0$ there exists $\beta>0$ such that

$$
(2[(k+1) / 2] \pi)^{\alpha} \leq \ell_{c, k}^{\beta} ; \quad \forall k \geq 1 .
$$

Then $A_{\alpha}^{-1}$ is Hilbert-Schmidt, $\inf \operatorname{Spec}\left(A_{\alpha}\right)>1$ and the $p$-norms are defined by

$$
|\xi|_{c, \alpha, p}^{2}=\left|A_{\alpha}^{p} \xi\right|_{c, \alpha}^{2}=\sum_{k=1}^{\infty} \ell_{c, k}^{2 p}\left|\left\langle\xi, J\left(e_{\alpha, k}\right)\right\rangle_{c, \alpha}\right|^{2}, \quad \xi \in H_{c, \alpha} .
$$

For $p \geq 0$ one defines

$$
\mathcal{N}_{c, \alpha, p}=\left\{\xi \in H_{c, \alpha}:|\xi|_{c, \alpha, p}<\infty\right\}
$$

and $\mathcal{N}_{c, \alpha,-p}$ to be the completion of $H_{c, \alpha}$ with respect to the norm $|\cdot|_{c, \alpha,-p}$. Thus we obtain a chain of Hilbert spaces $\left\{\mathcal{N}_{c, \alpha, p}: p \in \mathbb{R}\right\}$ and the corresponding triple:

$$
\mathcal{N}_{c, \alpha}:=\underset{p \rightarrow \infty}{\operatorname{proj} \lim } \mathcal{N}_{c, \alpha, p} \subset H_{c, \alpha} \subset \mathcal{N}_{c, \alpha}^{*}:=\operatorname{ind}_{p \rightarrow \infty} \lim \mathcal{N}_{c, \alpha,-p}
$$

which now depends on the triple $\left(H_{c, \alpha},\left\{e_{\alpha, k}\right\},\left\{\ell_{c, k}\right\}\right)$. 
Using the duality $\left\langle\mathcal{N}_{c, \alpha}^{*}, \mathcal{N}_{c, \alpha}\right\rangle$ the usual trace $\tau_{\alpha}$ on $H_{c, \alpha}$ is characterized by strong continuity and

$$
\left\langle\tau_{\alpha}, z \otimes w\right\rangle=\langle z, w\rangle
$$

can be considered as an element in the dual of $\mathcal{N}_{c, \alpha} \otimes \mathcal{N}_{c, \alpha}$ and in fact identified to

$$
\tau_{\alpha}=\sum_{k=1}^{\infty} e_{\alpha, k} \otimes e_{\alpha, k}
$$

More precisely one has the estimate.

Theorem 6.2. The trace $\tau_{\alpha}$ belongs to $\mathcal{N}_{c, \alpha,-1 / 2} \otimes \mathcal{N}_{c, \alpha,-1 / 2}$.

Proof. By definition we have

$$
\left|\tau_{\alpha}\right|_{c, \alpha,-1 / 2}^{2}=\sum_{k=1}^{\infty} \ell_{c, k}^{-2}\left|e_{\alpha, k} \otimes e_{\alpha, k}\right|_{c, \alpha}^{2}=\left\|A_{\alpha}^{-1}\right\|_{\mathrm{HS}}^{2}<\infty
$$

which implies the assertion.

Remark 6.2. Let $\alpha \in \mathbb{N}$. For $N \in \mathbb{N}$, let $p_{N}$ be the operator on $\mathcal{N}_{c, \alpha}^{*}$ given by

$$
p_{N} f=\sum_{k=1}^{N}\left\langle f, J\left(e_{\alpha, k}\right)\right\rangle_{c, \alpha} e_{\alpha, k} \quad \text { for } f \in \mathcal{N}_{c, \alpha}^{*} .
$$

Then, for any $f \in \mathcal{N}_{c, \alpha}$, we have $p_{N} f \in E^{*}$. For any $f \in \mathcal{N}_{c, \alpha} \cap E^{*}$, there exists some $p>1$ such that the $p$-norm $|f|_{-p}$ is estimated as follows:

$$
\begin{aligned}
|f|_{-p}^{2} & =\sum_{\nu=1}^{\infty} \ell_{\nu}^{-2 p}\left|\left\langle f, \bar{e}_{\nu}\right\rangle\right|^{2} \\
& \leq \frac{\alpha}{(2 \pi)^{\alpha-1}} \sum_{\nu=1}^{\infty}\left(2 \pi\left[\frac{\nu+1}{2}\right]\right)^{\alpha-1} \ell_{\nu}^{-2 p}\left(\sum_{k=1}^{\infty}\left|\left\langle f, J\left(e_{\alpha, k}\right)\right\rangle_{c, \alpha}\right|\right)^{2} \\
& \leq \frac{\alpha}{(2 \pi)^{\alpha-1}} \sum_{\nu=1}^{\infty} \ell_{\nu}^{-2(p-q)} \sum_{k=1}^{\infty} \ell_{c, k}^{2}\left|\left\langle f, J\left(e_{\alpha, k}\right)\right\rangle_{c, \alpha}\right|^{2} \sum_{k=1}^{\infty} \ell_{c, k}^{-2} \\
& =\frac{\alpha}{(2 \pi)^{\alpha-1}} \sum_{\nu=1}^{\infty} \ell_{\nu}^{-2(p-q)}|f|_{c, \alpha, 1}^{2} \sum_{k=1}^{\infty} \ell_{c, k}^{-2}<\infty
\end{aligned}
$$

for some $q>0$ such that $(2 \pi[(\nu+1) / 2])^{\alpha-1} \leq \ell_{\nu}^{2 q}$ for all $\nu \geq 1$. By this estimation we see that for any $f \in \mathcal{N}_{c, \alpha}$ the sequence $\left(p_{N} f\right)_{N=1}^{\infty}$ converges to $\widetilde{f}$ in $E^{*}$.

Let $\tilde{\mathcal{N}}_{c, \alpha}=\left\{\tilde{f}: f \in \mathcal{N}_{c, \alpha}\right\}$ with induced topology from $\mathcal{N}_{c, \alpha}$. Define the norm $|\widetilde{f}|_{c, \alpha, p}$ on $\widetilde{\mathcal{N}}_{c, \alpha}$ by $|\widetilde{f}|_{c, \alpha, p}=|f|_{c, \alpha, p}$ for any $f \in \mathcal{N}_{c, \alpha}$. Then $\mathcal{N}_{c, \alpha}$ is isomorphic to $\widetilde{\mathcal{N}}_{c, \alpha}$ in $E^{*}$ by norms $|\cdot|_{c, \alpha, p}, p \in \mathbb{R}$. This kind of embedding is discussed in detail in Ref. 11 by Harada. 


\section{Exotic HKT Spaces}

For each $\alpha \in \mathbb{N}$ and $p \in \mathbb{R}$ let $\Gamma\left(\mathcal{N}_{c, \alpha, p}\right)$ be the Fock space over the Hilbert space $\mathcal{N}_{c, \alpha, p}$, i.e.,

$$
\Gamma\left(\mathcal{N}_{c, \alpha, p}\right):=\left\{\phi=\left(f_{n}\right)_{n=0}^{\infty}: f_{n} \in \mathcal{N}_{c, \alpha, p}^{\widehat{\otimes} n},\|\phi\|_{c, \alpha, p}^{2}=\sum_{n=0}^{\infty} n !\left|f_{n}\right|_{c, \alpha, p}^{2}<\infty\right\} .
$$

Then by identifying $\Gamma\left(H_{c, \alpha}\right)$ with its dual space, we have a rigging of Fock spaces:

$$
\begin{aligned}
\left(\mathcal{N}_{c, \alpha}\right) & =\underset{p \rightarrow \infty}{\operatorname{proj} \lim } \Gamma\left(\mathcal{N}_{c, \alpha, p}\right) \subset \cdots \subset \Gamma\left(\mathcal{N}_{c, \alpha, p}\right) \subset \cdots \\
& \subset \Gamma\left(\mathcal{N}_{c, \alpha, 0}\right)=\Gamma\left(H_{c, \alpha}\right) \subset \Gamma\left(\mathcal{N}_{c, \alpha,-p}\right) \subset \cdots \\
& \subset\left(\mathcal{N}_{c, \alpha}\right)^{*}=\underset{p \rightarrow \infty}{\operatorname{ind} \lim } \Gamma\left(\mathcal{N}_{c, \alpha,-p}\right) .
\end{aligned}
$$

The canonical $\mathbb{C}$-bilinear form on $\left(\mathcal{N}_{c, \alpha}\right)^{*} \times\left(\mathcal{N}_{c, \alpha}\right)$ is defined, for any $\Phi=\left(F_{n}\right) \in$ $\left(\mathcal{N}_{c, \alpha}\right)^{*}$ and $\phi=\left(f_{n}\right) \in\left(\mathcal{N}_{c, \alpha}\right)$, by

$$
\langle\langle\Phi, \phi\rangle\rangle_{c, \alpha}=\sum_{n=0}^{\infty} n !\left\langle F_{n}, f_{n}\right\rangle_{c, \alpha} .
$$

The exponential vectors $\phi_{\xi}$ are defined as in (2.16). The $S_{c, \alpha}$-transform of an element $\Phi \in\left(\mathcal{N}_{c, \alpha}\right)^{*}$ is defined by

$$
S_{c, \alpha} \Phi(\xi)=\left\langle\left\langle\Phi, \phi_{\xi}\right\rangle_{c, \alpha}, \quad \xi \in \mathcal{N}_{c, \alpha} .\right.
$$

Then since $\left(\mathcal{N}_{c, \alpha}\right)$ is a nuclear space, as a similar result to Theorem 2.1 we prove that a $\mathbb{C}$-valued function $F$ on $\mathcal{N}_{c, \alpha}$ is the $S_{c, \alpha}$-transform of an element in $\left(\mathcal{N}_{c, \alpha}\right)^{*}$ if and only if $F$ is Gâteaux entire, and there exist constants $C, K \geq 0$ and $p \geq 0$ such that

$$
|F(\xi)| \leq C \exp \left(K|\xi|_{c, \alpha, p}^{2}\right), \quad \xi \in \mathcal{N}_{c, \alpha} .
$$

Using similar methods in Lemmas 3.1 and 3.2 , we have the following:

Lemma 7.1. For any $\alpha \in \mathbb{N}$, the operator $\partial_{\alpha}$ is an isomorphism from $\mathcal{N}_{c, 2 \alpha+1}$ onto itself.

Lemma 7.2. For any $\alpha \in \mathbb{N}$, the operator $\partial_{\alpha}$ is an isomorphism from $\mathcal{N}_{c, 2 \alpha+1}^{*}$ onto itself.

Remark 7.1. The Bochner-Minlos Theorem implies the existence of a probability measure $\mu_{c, \alpha}$ on $\mathcal{N}_{c, \alpha, \mathbb{R}}^{*}$ such that

$$
\int_{\mathcal{N}_{c, \alpha, \mathbb{R}}^{*}} e^{i\langle x, \xi\rangle_{c, \alpha}} d \mu_{c, \alpha}(x)=e^{-\frac{1}{2}\langle\xi, \xi\rangle_{c, \alpha}} ; \quad \xi \in \mathcal{N}_{c, \alpha, \mathbb{R}}
$$

The standard triple consisting of white noise distributions obtained from (7.1) through the Wiener-Itô-Segal isomorphism is denoted also by

$$
\left(\mathcal{N}_{c, \alpha}\right) \subset \Gamma\left(H_{c, \alpha}\right) \cong L^{2}\left(\mathcal{N}_{c, \alpha, \mathbb{R}}^{*}, \mu_{c, \alpha}\right) \subset\left(\mathcal{N}_{c, \alpha}\right)^{*} .
$$

We call $\left(\mathcal{N}_{c, \alpha}\right)^{*}$ the exotic Hida-Kubo-Takenaka (HKT) space of order $\alpha$. 


\section{Exotic Laplacians on Exotic HKT Spaces}

From Remark 6.2, we introduce an isomorphism $i_{\alpha}$ from $\left(\mathcal{N}_{c, \alpha}\right)$ onto $\left(\widetilde{\mathcal{N}}_{c, \alpha}\right)$ given by

$$
i_{\alpha}\left(\left(f_{n}\right)\right)=\left(\widetilde{f_{n}}\right)
$$

for $\left(f_{n}\right) \in\left(\mathcal{N}_{c, \alpha}\right)$, Then we have the following.

Theorem 8.1. Any element $\varphi \in\left(\mathcal{N}_{c, \alpha}\right)$ is in $\operatorname{Dom}\left(\Delta_{c, \alpha}\right)$. Moreover, if $\varphi=$ $\left(f_{n}\right)_{n=0}^{\infty}$, then we have

$$
\Delta_{c, \alpha} i_{\alpha} \varphi=\left((n+2)(n+1) \tau_{\alpha} \widehat{\widehat{\otimes}^{2} f_{n+2}}\right) .
$$

Proof. For the proof, we refer to Ref. 16.

By Theorem 8.1, we define an operator $\bar{\Delta}_{c, \alpha}$ on $\left(\mathcal{N}_{c, \alpha}\right)$ by $\bar{\Delta}_{c, \alpha}=i_{\alpha}^{-1} \Delta_{c, \alpha} i_{\alpha}$. Then this operator is a continuous linear operator from $\left(\mathcal{N}_{c, \alpha}\right)$ into itself. For each $t \in \mathbb{R}$ there exists a unique operator $G_{t} \in \mathcal{L}\left(\left(\mathcal{N}_{c, \alpha}\right),\left(\mathcal{N}_{c, \alpha}\right)\right)$ such that

$$
\left\langle\left\langle G_{t} \phi_{\xi}, \phi_{\eta}\right\rangle\right\rangle_{c, \alpha}=e^{\frac{t}{2}\left\langle\tau_{\alpha}, \xi \otimes \xi\right\rangle_{c, \alpha}+\langle\xi, \eta\rangle_{c, \alpha}}, \quad \xi, \eta \in \mathcal{N}_{c, \alpha} .
$$

In fact, for any $\phi=\left(f_{n}\right) \in\left(\mathcal{N}_{c, \alpha}\right), G_{t} \phi$ is given by

$$
G_{t} \phi=\left(\sum_{m=0}^{\infty} \frac{(n+2 m) !}{n ! m !}\left(\frac{t}{2}\right)^{m}\left(\tau_{\alpha}^{\otimes m} \widehat{\otimes}_{2 m} f_{n+2 m}\right)\right) .
$$

Theorem 8.2. $\left\{G_{t}: t \in \mathbb{R}\right\}$ becomes a regular one-parameter group of operators acting on $\left(\mathcal{N}_{c, \alpha}\right)$ with infinitesimal generator $\frac{1}{2} \bar{\Delta}_{c, \alpha}$.

The proof is a simple modification of the proof of Theorem 4.3 in Ref. 16.

Remark 8.1. By Theorem 8.2 and Corollary 5.1 we have

$$
G_{t}=i_{\alpha}^{-1} \Gamma\left(p^{\alpha}\right) e^{\frac{t}{2} \Delta_{L}} \Gamma\left(p^{\alpha}\right)^{-1} i_{\alpha} \quad \text { on }\left(\mathcal{N}_{c, \alpha}\right) .
$$

\section{Stochastic Processes Generated by Exotic Laplacians}

In this section, we study infinite dimensional stochastic processes generated by the exotic Laplacians.

Let $\left\{\mathbf{X}_{t}: t \geq 0\right\}$ be a $\left(\mathcal{N}_{c, \alpha}\right)$-valued stochastic process. Then we can write the process in the form $\mathbf{X}_{t}=\left(\mathbf{X}_{t, n}\right)$. The expectation $E\left[\mathbf{X}_{t}\right]$ of $\mathbf{X}_{t}$, if it exists in $\left(\mathcal{N}_{c, \alpha}\right)$, is given by

$$
E\left[\mathbf{X}_{t}\right]=\left(E\left[\mathbf{X}_{t, n}\right]\right)
$$

For $\eta \in \mathcal{N}_{c, \alpha}$ let $\mathbf{T}_{\eta}$ be the translation operator defined on $\left(\mathcal{N}_{c, \alpha}\right)^{*}$ by

$$
\mathbf{T}_{\eta} \Phi=\sum_{k=0}^{\infty} \frac{1}{k !} D_{\eta}^{k} \Phi ; \quad \Phi \in\left(\mathcal{N}_{c, \alpha}\right)^{*}
$$


where $D_{\eta}$ is defined by $D_{\eta} \Phi=S_{c, \alpha}^{-1}\left(\widetilde{D}_{\eta} S_{c, \alpha} \Phi\right)$. Then we have

$$
D_{\eta} \Phi=\left((n+1)\left\langle F_{n+1}, \eta\right\rangle_{c, \alpha}\right) ; \quad \Phi=\left(F_{n}\right) \in\left(\mathcal{N}_{c, \alpha}\right)^{*}
$$

and

$$
\mathbf{T}_{\eta} \Phi=\left(\sum_{k=0}^{\infty} \frac{(n+k) !}{n ! k !}\left\langle F_{n+k}, \eta^{\otimes k}\right\rangle_{c, \alpha}\right) .
$$

Therefore, for given $z \in \mathcal{N}_{c, \alpha}^{*}$ and $\Phi \in\left(\mathcal{N}_{c, \alpha}\right)^{*}$, it is natural to define $\mathbf{T}_{z} \Phi$ by (9.1) whenever the right-hand side of $(9.1)$ is well defined as an element in $\left(\mathcal{N}_{c, \alpha}\right)^{*}$.

Proposition 9.1. For all $z \in \mathcal{N}_{c, \alpha}^{*}$, the operator $\mathbf{T}_{z}$ is in $\mathcal{L}\left(\left(\mathcal{N}_{c, \alpha}\right),\left(\mathcal{N}_{c, \alpha}\right)\right)$. Furthermore, for any $p \geq 0, q>0$ with $|z|_{c, \alpha,-(p+q)}<\infty$, it holds that

$$
\left\|\mathbf{T}_{z} \phi\right\|_{c, \alpha, p} \leq \frac{\|\phi\|_{c, \alpha, p+q}}{\left(1-\ell_{c, 1}^{-2 q}\right)} \exp \left(\frac{|z|_{c, \alpha,-(p+q)}^{2}}{2\left(1-\ell_{c, 1}^{-2 q}\right)}\right), \quad \phi \in\left(\mathcal{N}_{c, \alpha}\right) .
$$

Proof. The proof is a simple modification of the proof of Theorem 4.2.3 in Ref. 24.

Let $\left\{\left\{B_{k}(t): t \geq 0\right\}\right\}_{k=1}^{\infty}$ be an infinite sequence of independent one-dimensional Brownian motions and $\left\{\mathbf{B}_{\alpha}(t): t \geq 0\right\}$ the infinite dimensional stochastic process defined by

$$
\mathbf{B}_{\alpha}(t)=\sum_{k=1}^{\infty} B_{k}(t) e_{\alpha, k} ; \quad t \geq 0 .
$$

Lemma 9.1. For all $t \geq 0$ we have $\mathbf{B}_{\alpha}(t) \in \mathcal{N}_{c, \alpha}^{*}$ (a.e.)

Proof. By definition, we can check that, for any $t \geq 0$ and $p \geq 1$ one has

$$
\begin{aligned}
E\left[\left|\mathbf{B}_{\alpha}(t)\right|_{c, \alpha,-p}^{2}\right] & =E\left[\sum_{k=1}^{\infty} \ell_{c, k}^{-2 p}\left|\left\langle\mathbf{B}_{\alpha}(t), J\left(e_{\alpha, k}\right)\right\rangle_{c, \alpha}\right|^{2}\right] \\
& =\sum_{k=1}^{\infty} \ell_{c, k}^{-2 p} E\left[\left|B_{k}(t)\right|^{2}\right] \\
& =t \sum_{k=1}^{\infty} \ell_{c, k}^{-2 p}<\infty,
\end{aligned}
$$

which implies the assertion.

Theorem 9.1. Let $\phi \in\left(\mathcal{N}_{c, \alpha}\right)$. Then the equality

$$
G_{t} \phi=E\left[\mathbf{T}_{\mathbf{B}_{\alpha}(t)} \phi\right]
$$

holds for $t \geq 0$. 
Proof. Let $\phi=\left(f_{n}\right) \in\left(\mathcal{N}_{c, \alpha}\right)$. Then by (9.1) we have

$$
\mathbf{T}_{\mathbf{B}_{\alpha}(t)} \phi=\left(\sum_{k=0}^{\infty} \frac{(n+k) !}{n ! k !}\left\langle f_{n+k}, \mathbf{B}_{\alpha}(t)^{\otimes k}\right\rangle_{c, \alpha}\right)
$$

and by (9.2) we have

$$
\left\langle f_{n+k}, \mathbf{B}_{\alpha}(t)^{\otimes k}\right\rangle_{c, \alpha}=\sum_{\ell_{1}, \ldots, \ell_{k}=1}^{\infty}\left[\prod_{j=1}^{k} B_{\ell_{j}}(t)\right]\left\langle f_{n+k}, \bigotimes_{j=1}^{k} e_{\alpha, \ell_{j}}\right\rangle_{c, \alpha} .
$$

Therefore, we have

$$
\begin{aligned}
S_{c, \alpha} & {\left[E\left[\mathbf{T}_{\mathbf{B}_{\alpha}(t)} \phi\right]\right](\xi) } \\
& =\sum_{m=0}^{\infty} \frac{t^{m}}{2^{m} m !} \sum_{n=0}^{\infty} \sum_{j=1}^{m} \frac{(n+2 m) !}{j !}\left\langle f_{n+2 m} \sum_{\begin{array}{c}
\ell_{1}, \ldots, \ell_{j} \\
\text { all different }
\end{array}}\left(\sum_{\nu=1}^{j} e_{\alpha, \ell_{\nu}}^{\otimes 2}\right)^{\otimes m} \widehat{\otimes} \xi^{\otimes n}\right\rangle_{c, \alpha} \\
& =\sum_{n=0}^{\infty} \sum_{m=0}^{\infty} \frac{(n+2 m) !}{m !}\left(\frac{t}{2}\right)^{m}\left\langle f_{n+2 m},\left(\sum_{\ell=1}^{\infty} e_{\alpha, \ell}^{\otimes 2}\right)^{\otimes m} \widehat{\otimes} \xi^{\otimes n}\right\rangle_{c, \alpha} \\
& =\sum_{n=0}^{\infty} \sum_{m=0}^{\infty} \frac{(n+2 m) !}{m !}\left(\frac{t}{2}\right)^{m}\left\langle\tau_{\alpha}^{\otimes m} \hat{\otimes}_{2 m} f_{n+2 m}, \xi^{\otimes n}\right\rangle_{c, \alpha} .
\end{aligned}
$$

This implies

$$
\begin{aligned}
E\left[\mathbf{T}_{\mathbf{B}_{\alpha}(t)} \phi\right] & =\left(\sum_{m=0}^{\infty} \frac{(n+2 m) !}{n ! m !}\left(\frac{t}{2}\right)^{m}\left(\tau_{\alpha}^{\otimes m} \widehat{\otimes}_{2 m} f_{n+2 m}\right)\right) \\
& =G_{t} \phi
\end{aligned}
$$

which gives the proof.

By Theorem 9.1, we can consider $\left\{\mathbf{B}_{\alpha}(t)\right\}_{t \geq 0}$ as a stochastic process generated by the extended exotic Laplacian $\frac{1}{2} \bar{\Delta}_{c, \alpha}$.

Theorem 9.2. For $\alpha \geq 0$ the $\mathcal{N}_{c, 2 \alpha+1}^{*}$-valued stochastic process

$$
\mathbf{B}_{1}(t)^{(\alpha)}=\sum_{n=1}^{\infty} B_{n}(t) e_{1, n}^{(\alpha)}
$$

is generated by $\frac{\gamma}{2} \bar{\Delta}_{c, 2 \alpha+1}, \gamma=\frac{(2 \pi)^{2 \alpha}}{2 \alpha+1}$, acting on $\left(\mathcal{N}_{c, 2 \alpha+1}\right)$, where $\mathbf{B}_{1}(t)^{(\alpha)}$ is the $\alpha$ th derivative of the $\mathcal{N}_{c, 1}^{*}$-valued stochastic process $\mathbf{B}_{1}(t)$. 


\section{References}

1. L. Accardi, A. Barhoumi and H. Ouerdiane, A quantum approach to Laplace operators, Infin. Dimen. Anal. Quantum Probab. Rel. Top. 9(2) (2006) 215-248.

2. L. Accardi, P. Gibilisco and I. V. Volovich, The Lévy Laplacian and the Yang-Mills equations, Russ. J. Math. Phys. 2 (1994) 235-250.

3. L. Accardi and O. G. Smolyanov, On Laplacians and traces, in Conferenze del Seminario di Matematica dell'Universit'a di Bari, N. 250 (1993).

4. L. Accardi and O. G. Smolyanov, The trace formulae for Lévy-Gaussian measures and their applications, in Mathematical Approach to Fluctuations, Vol. II, ed. T. Hida (World Scientific, Singapore, 1995), pp. 31-47.

5. L. Accardi and O. G. Smolyanov, Generalized Lévy Laplacians and Cesàro means, Doklady Akademii Nauk 424 (2009) 583-587.

6. L. Accardi, U. C. Ji and K. Saitô, Exotic Laplacians and associated stochastic processes, Infin. Dimen. Anal. Quantum Probab. Rel. Top. 12(1) (2009) 1-19.

7. L. Accardi, U. C. Ji and K. Saitô, Exotic Laplacians and derivatives of white noise, Infin. Dimen. Anal. Quantum Probab. Rel. Top. 14(1) (2011) 1-14.

8. D. M. Chung, U. C. Ji and K. Saitô, Cauchy problems associated with the Lévy Laplacian in white noise analysis, Infin. Dimen. Anal. Quantum Probab. Rel. Top. 2(1) (1999) 131-153.

9. D. M. Chung, U. C. Ji and K. Saitô, Notes on a $C_{0}$-group generated by the Lévy Laplacian, Proc. Amer. Math. Soc. 130 (2001) 1197-1206.

10. I. M. Gel'fand and N. Ya. Vilenkin, Generalized Functions, Vol. 4 (Academic Press, 1964).

11. K. Harada, Infinite dimensional Laplacians and associated partial differential equations, PhD thesis, Nagoya University (2011).

12. T. Hida, Analysis of Brownian functionals, in Carleton Math. Lect. Notes, No. 13 (Carleton University, Ottawa, 1975).

13. T. Hida, H.-H. Kuo, J. Potthoff and L. Streit, White Noise: An Infinite Dimensional Calculus (Kluwer Academic Publishers, 1993).

14. E. Hille and R. S. Phillips, Functional analysis and semi-groups, in Amer. Math. Soc. Colloquium Publications 31 (1957).

15. U. C. Ji, Integral kernel operators on regular generalized white noise functions, Bull. Korean Math. Soc. 37(3) (2000) 601-618.

16. U. C. Ji and K. Saitô, A similarity between the gross Laplacian and the Lévy Laplacian, Infin. Dimen. Anal. Quantum Probab. Rel. Top. 10(2) (2007) 261-276.

17. I. Kubo and S. Takenaka, Calculus on Gaussian white noise I-IV, Proc. Japan Acad. 56A (1980) 376-380, 56A (1980) 411-416, 57A (1981) 433-436, 58A (1982) $186-189$.

18. H.-H. Kuo, White Noise Distribution Theory (CRC Press, 1996).

19. H.-H. Kuo, N. Obata and K. Saitô, Lévy Laplacian of generalized functions on a nuclear space, J. Funct. Anal. 94 (1990) 74-92.

20. H.-H. Kuo, N. Obata and K. Saitô, Diagonalization of the Lévy Laplacian and related stable processes, Infin. Dimen. Anal. Quantum Probab. Rel. Top. 5(3) (2002) 317-331.

21. P. Lévy, Lecons d'Analyse Fonctionnelle (Gauthier-Villars, Paris, 1922).

22. P. Lévy, Problèms Concrets d'Analyse Fonctionnelle (Gauthier-Villars, Paris, 1951).

23. N. Obata, A characterization of the Lévy Laplacian in terms of infinite dimensional rotation groups, Nagoya Math. J. 118 (1990) 111-132.

24. N. Obata, White noise calculus and Fock space, in Lect. Notes in Math. Vol. 1577 (Springer-Verlag, Berlin, Heidelberg, 1994). 
25. J. Potthoff and L. Streit, A characterization of Hida distributions, J. Funct. Anal. 101 (1991) 212-229.

26. K. Saitô, Itô's formula and Lévy Laplacian I and II, Nagoya Math. J. 108 (1987) 67-76, 123 (1991) 153-169.

27. K. Saitô, A $\left(C_{0}\right)$-group generated by the Lévy Laplacian II, Infin. Dimen. Anal. Quantum Probab. Rel. Top. 1(3) (1998) 425-437.

28. K. Saitô, A stochastic process generated by the Lévy Laplacian, Acta Appl. Math. 63 (2000) 363-373. 